\title{
Compact Riemannian Manifolds with Homogeneous Geodesics*
}

\author{
Dmitriน V. ALEKSEEVSKY ${ }^{\dagger}$ and Yuriu G. NIKONOROV ${ }^{\ddagger}$ \\ $\dagger$ School of Mathematics and Maxwell Institute for Mathematical Studies, Edinburgh University, \\ Edinburgh EH9 3JZ, United Kingdom \\ E-mail:D.Aleksee@ed.ac.uk \\ $¥$ Volgodonsk Institute of Service (branch) of South Russian State University of Economics \\ and Service, 16 Mira Ave., Volgodonsk, Rostov region, 347386, Russia \\ E-mail: nikonorov2006@mail.ru
}

Received April 22, 2009, in final form September 20, 2009; Published online September 30, 2009

doi:10.3842/SIGMA.2009.093

\begin{abstract}
A homogeneous Riemannian space $(M=G / H, g)$ is called a geodesic orbit space (shortly, GO-space) if any geodesic is an orbit of one-parameter subgroup of the isometry group $G$. We study the structure of compact GO-spaces and give some sufficient conditions for existence and non-existence of an invariant metric $g$ with homogeneous geodesics on a homogeneous space of a compact Lie group $G$. We give a classification of compact simply connected GO-spaces $(M=G / H, g)$ of positive Euler characteristic. If the group $G$ is simple and the metric $g$ does not come from a bi-invariant metric of $G$, then $M$ is one of the flag manifolds $M_{1}=S O(2 n+1) / U(n)$ or $M_{2}=S p(n) / U(1) \cdot S p(n-1)$ and $g$ is any invariant metric on $M$ which depends on two real parameters. In both cases, there exists unique (up to a scaling) symmetric metric $g_{0}$ such that $\left(M, g_{0}\right)$ is the symmetric space $M=S O(2 n+2) / U(n+1)$ or, respectively, $\mathbb{C} P^{2 n-1}$. The manifolds $M_{1}, M_{2}$ are weakly symmetric spaces.
\end{abstract}

Key words: homogeneous spaces, weakly symmetric spaces, homogeneous spaces of positive Euler characteristic, geodesic orbit spaces, normal homogeneous Riemannian manifolds, geodesics

2000 Mathematics Subject Classification: 53C20; 53C25; 53C35

\section{Introduction}

A Riemannian manifold $(M, g)$ is called a manifold with homogeneous geodesics or geodesic orbit manifold (shortly, GO-manifold) if all its geodesic are orbits of one-parameter groups of isometries of $(M, g)$. Such manifold is a homogeneous manifold and can be identified with a coset space $M=G / H$ of a transitive Lie group $G$ of isometries. A Riemannian homogeneous space $\left(M=G / H, g^{M}\right)$ of a group $G$ is called a space with homogeneous geodesics (or geodesic orbit space, shortly, GO-space) if any geodesic is an orbit of a one-parameter subgroup of the group $G$. This terminology was introduced by O. Kowalski and L. Vanhecke in [20], who initiated a systematic study of such spaces.

Recall that homogeneous geodesics correspond to "relative equilibria" of the geodesic flow, considered as a hamiltonian system on the cotangent bundle. Due to this, GO-manifolds can be characterized as Riemannian manifolds such that all integral curves of the geodesic flow are relative equilibria.

\footnotetext{
*This paper is a contribution to the Special Issue "Élie Cartan and Differential Geometry". The full collection is available at http://www.emis.de/journals/SIGMA/Cartan.html
} 
GO-spaces may be considered as a natural generalization of symmetric spaces, classified by É. Cartan [10]. Indeed, a simply connected symmetric space can be defined as a Riemannian manifold $(M, g)$ such that any geodesic $\gamma \subset M$ is an orbit of one-parameter group $g_{t}$ of transvections, that is one-parameter group of isometries which preserves $\gamma$ and induces the parallel transport along $\gamma$. If we remove the assumption that $g_{t}$ induces the parallel transport, we get the notion of a GO-space.

The class of GO-spaces is much larger then the class of symmetric spaces. Any homogeneous space $M=G / H$ of a compact Lie group $G$ admits a metric $g^{M}$ such that $\left(M, g^{M}\right)$ is a GO-space. It is sufficient to take the metric $g^{M}$ which is induced with a bi-invariant Riemannian metric $g$ on the Lie group $G$ such that $(G, g) \rightarrow\left(M=G / H, g^{M}\right)$ is a Riemannian submersion with totally geodesic fibres. Such GO-space $\left(M=G / H, g^{M}\right)$ is called a normal homogeneous space.

More generally, any naturally reductive manifold is a geodesic orbit manifold. Recall that a Riemannian manifold $\left(M, g^{M}\right)$ is called naturally reductive if it admits a transitive Lie group $G$ of isometries with a bi-invariant pseudo-Riemannian metric $g$, which induces the metric $g^{M}$ on $M=G / H$, see $[18,8]$. The first example of non naturally reductive GO-manifold had been constructed by A. Kaplan [16]. An important class of GO-spaces consists of weakly symmetric spaces, introduced by A. Selberg [22]. A homogeneous Riemannian space $\left(M=G / H, g^{M}\right)$ is a weakly symmetric space if any two points $p, q \in M$ can be interchanged by an isometry $a \in G$. This property does not depend on the particular invariant metric $g^{M}$. Weakly symmetric spaces $M=G / H$ have many interesting properties (for example, the algebra of $G$-invariant differential operators on $M$ is commutative, the representation of $G$ in the space $L^{2}(M)$ of function is multiplicity free, the algebra of $G$-invariant Hamiltonians on $T^{*} M$ with respect to Poisson bracket is commutative) and are closely related with spherical spaces, commutative spaces and Gelfand pairs etc., see the book by J.A. Wolf [26]. The classification of weakly symmetric reductive homogeneous spaces was given by O.S. Yakimova [28], see also [26].

In [20], O. Kowalski and L. Vanhecke classified all GO-spaces of dimension $\leq 6$. C. Gordon [14] reduced the classification of GO-spaces to the classification of GO-metrics on nilmanifolds, compact GO-spaces and non-compact GO-spaces of non-compact semisimple Lie group. She described GO-metrics on nilmanifolds. They exist only on two-step nilponent nilmanifolds. She also presented some constructions of GO-metrics on homogeneous compact manifolds and non compact manifolds of a semisimple group.

Many interesting results about GO-spaces one can find in [7, 12, 27, 23, 24], where there are also extensive references.

Natural generalizations of normal homogeneous Riemannian manifolds are $\delta$-homogeneous Riemannian manifolds, studied in $[3,4,5]$. Note that the class of $\delta$-homogeneous Riemannian manifolds is a proper subclass of the class of geodesic orbit spaces with non-negative sectional curvature (see the quoted papers for further properties of $\delta$-homogeneous Riemannian manifolds).

In [1], a classification of non-normal invariant GO-metrics on flag manifolds $M=G / H$ was given. The problem reduces to the case when the (compact) group $G$ is simple. There exist only two series of flag manifolds of a simple group which admit such metric, namely weakly symmetric spaces $M_{1}=S O(2 n+1) / U(n)$ and $M_{2}=S p(n) / U(1) \cdot S p(n-1)$, equipped with any (non-normal) invariant metric (which depends on two real parameters). Moreover, there exists unique (up to a scaling) invariant metric $g_{0}$, such that the Riemannian manifolds $\left(M_{i}, g_{0}\right)$ are isometric to the symmetric spaces $S O(2 n+2) / U(n+1)$ and $\mathbf{C} P^{2 n-1}=S U(2 n) / U(2 n-1)$, respectively.

The main goal of this paper is a generalization of this result to the case of compact homogeneous manifolds of positive Euler characteristic. We prove that the weakly symmetric manifolds $M_{1}, M_{2}$ exhaust all simply connected compact irreducible Riemannian non-normal GO-manifolds of positive Euler characteristic. 
We indicate now the idea of the proof. Let $\left(M=G / H, g^{M}\right)$ be a compact irreducible nonnormal GO-space of positive Euler characteristic. Then the stability subgroup $H$ has maximal rank, which implies that $G$ is simple. We prove that there is rank 2 regular simple subgroup $G^{\prime}$ of $G$ (associated with a rank 2 subsystem $R^{\prime}$ of the root system $R$ of the Lie algebra $\mathfrak{g}=\operatorname{Lie}(G)$ ) such that the orbit $M^{\prime}=G^{\prime} o=G^{\prime} / H^{\prime}$ of the point $o=e H \in M$ (with the induced metric) is a non-normal GO-manifold. Using [1,3], we prove that the only such manifold $M^{\prime}$ is $S U(5) / U(2)$. This implies that the root system $R$ is not simply-laced and admits a "special" decomposition $R=R_{0} \cup R_{1} \cup R_{2}$ into a disjoint union of three subsets, which satisfies some properties. We determine all such special decompositions of irreducible root systems and show that only root systems of type $B_{n}$ and $C_{n}$ admit special decomposition and associated homogeneous manifolds are $M_{1}$ and $M_{2}$.

The structure of the paper is the following. We fix notations and recall basic definitions in Section 2. Some standard facts about totally geodesic submanifolds of a homogeneous Riemannian spaces are collected in Section 3. We discuss some properties of compact GO-spaces in Section 4. These results are used in Section 5 to derive sufficient conditions for existence and non-existence of a non-normal GO-metric on a homogeneous manifold of a compact group. Section 6 is devoted to classification of compact GO-spaces with positive Euler characteristic.

\section{Preliminaries and notations}

Let $M=G / H$ be a homogeneous space of a compact connected Lie group $G$. We will denote by $b=\langle\cdot, \cdot\rangle$ a fixed $\operatorname{Ad}_{G}$-invariant Euclidean metric on the Lie algebra $\mathfrak{g}$ of $G$ (for example, the minus Killing form if $G$ is semisimple) and by

$$
\mathfrak{g}=\mathfrak{h}+\mathfrak{m}
$$

the associated $b$-orthogonal reductive decomposition, where $\mathfrak{h}=\operatorname{Lie}(H)$. An invariant Riemannian metric $g^{M}$ on $M$ is determined by an $\operatorname{Ad}_{H}$-invariant Euclidean metric $g=(\cdot, \cdot)$ on the space $\mathfrak{m}$ which is identified with the tangent space $T_{o} M$ at the initial point $o=e H$.

If $\mathfrak{p}$ is a subspace of $\mathfrak{m}$, we will denote by $X_{\mathfrak{p}}$ the $b$-orthogonal projection of a vector $X \in \mathfrak{g}$ onto $\mathfrak{p}$, by $b_{\mathfrak{p}}$ the restriction of the symmetric bilinear form to $\mathfrak{p}$ and by $A^{\mathfrak{p}}=\operatorname{pr}_{\mathfrak{p}} \circ A \circ \mathrm{pr}_{\mathfrak{p}}$ the projection of an endomorphism $A$ to $\mathfrak{p}$. If $g$ is a $\operatorname{Ad}_{H}$-invariant metric, the quotient

$$
A=b_{\mathfrak{m}}^{-1} \circ g
$$

is an $\operatorname{Ad}_{H}$-equivariant symmetric positively defined endomorphism on $\mathfrak{m}$, which we call the metric endomorphism. Conversely, any such equivariant positively defined endomorphism $A$ of $\mathfrak{m}$ defines an invariant metric $g=b \circ A=b(A \cdot, \cdot)$ on $\mathfrak{m}$, hence an invariant Riemannian metric $g^{M}$ on $M$.

Lemma 1. Let $\left(M=G / H, g^{M}\right)$ be a compact homogeneous Riemannian space with metric endomorphism $A$ and

$$
\mathfrak{m}=\mathfrak{m}_{1} \oplus \mathfrak{m}_{2} \oplus \cdots \oplus \mathfrak{m}_{k}
$$

the A-eigenspace decomposition such that $\left.A\right|_{\mathfrak{m}_{i}}=\lambda_{i} \cdot \mathbf{1}_{\mathfrak{m}_{i}}$. Then

$$
\left(\mathfrak{m}_{i}, \mathfrak{m}_{j}\right)=\left\langle\mathfrak{m}_{i}, \mathfrak{m}_{j}\right\rangle=0
$$

and $\operatorname{Ad}_{H}$-modules $\mathfrak{m}_{i}$ satisfy $\left[\mathfrak{m}_{i}, \mathfrak{m}_{j}\right] \subset \mathfrak{m}$ for $i \neq j$. 
Proof. Since $A$ commute with $\mathrm{Ad}_{H}$, eigenspaces $\mathfrak{m}_{i}$ are $\operatorname{Ad}_{H}$-invariants and for $X \in \mathfrak{m}_{i}, Y \in \mathfrak{m}_{j}$, $i \neq j$, we get

$$
\lambda_{i}\langle X, Y\rangle=\langle A X, Y\rangle=(X, Y)=\langle X, A Y\rangle=\lambda_{j}\langle X, Y\rangle .
$$

This implies (3). The inclusion $\left[\mathfrak{m}_{i}, \mathfrak{m}_{j}\right] \subset \mathfrak{m}$ follows from the fact that $\mathfrak{m}_{j}$ is $\operatorname{Ad}_{H}$-invariant and $\left\langle\left[\mathfrak{m}_{i}, \mathfrak{m}_{j}\right], \mathfrak{h}\right\rangle=\left\langle\mathfrak{m}_{i},\left[\mathfrak{m}_{j}, \mathfrak{h}\right]\right\rangle=0$.

For any subspace $\mathfrak{p} \subset \mathfrak{m}$ we will denote by $\mathfrak{p}^{\perp}$ its orthogonal complement with respect to the metric $g$ and by $\mathbf{1}_{\mathfrak{p}}$ the identity operator on $\mathfrak{p}$.

Recall that $\operatorname{Ad}_{H}$-submodules $\mathfrak{p}, \mathfrak{q}$ are called disjoint if they have no non-zero equivalent submodules. If $\mathrm{Ad}_{H}$-module $\mathfrak{m}$ is decomposed into a direct sum

$$
\mathfrak{m}=\mathfrak{m}_{1}+\cdots+\mathfrak{m}_{k}
$$

of disjoint submodules, then any $\operatorname{Ad}_{H}$-invariant metric $g$ and associated metric endomorphism $A$ have the form

$$
g=g_{\mathfrak{m}_{1}} \oplus \cdots \oplus g_{\mathfrak{m}_{k}}, \quad A=A^{\mathfrak{m}_{1}} \oplus \cdots \oplus A^{\mathfrak{m}_{k}} .
$$

Let $\left(M=G / H, g^{M}\right)$ be a compact homogeneous Riemannian space with the reductive decomposition (1) and metric endomorphism $A \in \operatorname{End}(\mathfrak{m})$.

We identify elements $X, Y \in \mathfrak{g}$ with Killing vector fields on $M$. Then the covariant derivative $\nabla_{X} Y$ at the point $o=e H$ is given by

$$
\nabla_{X} Y(o)=-\frac{1}{2}[X, Y]_{\mathfrak{m}}+U\left(X_{\mathfrak{m}}, Y_{\mathfrak{m}}\right)
$$

where the bilinear symmetric map $U: \mathfrak{m} \times \mathfrak{m} \rightarrow \mathfrak{m}$ is given by

$$
2(U(X, Y), Z)=\left(\operatorname{ad}_{Z}^{\mathfrak{m}} X, Y\right)+\left(X, \operatorname{ad}_{Z}^{\mathfrak{m}} Y\right)
$$

for any $X, Y, Z \in \mathfrak{m}$ and $X_{\mathfrak{m}}$ is the $\mathfrak{m}$-part of a vector $X \in \mathfrak{g}[8]$.

Definition 1. A homogeneous Riemannian space $\left(M=G / H, g^{M}\right)$ is called a space with homogeneous geodesics shortly, GO-space if any geodesic $\gamma$ of $M$ is an orbit of 1-parameter subgroup of $G$. The invariant metric $g^{M}$ is called GO-metric.

If $G$ is the full isometry group, then GO-space is called a manifold with homogeneous geodesics or GO-manifold.

Definition 2. A GO-space $\left(M=G / H, g^{M}\right)$ of a simple compact Lie group $G$ is called a proper GO-space if the metric $g^{M}$ is not $G$-normal, i.e. the metric endomorphism $A$ is not a scalar operator.

Lemma 2 ([1]). A compact homogeneous Riemannian space $\left(M=G / H, g^{M}\right)$ with the reductive decomposition (1) and metric endomorphism $A$ is GO-space if and only if for any $X \in \mathfrak{m}$ there is $H_{X} \in \mathfrak{h}$ such that one of the following equivalent conditions holds:

i) $\left[H_{X}+X, A(X)\right] \in \mathfrak{h}$;

ii) $\left(\left[H_{X}+X, Y\right]_{\mathfrak{m}}, X\right)=0$ for all $Y \in \mathfrak{m}$.

This lemma shows that the property to be GO-space depends only on the reductive decomposition (1) and the Euclidean metric $g$ on $\mathfrak{m}$. In other words, if $\left(M=G / H, g^{M}\right)$ is a GO-space, then any locally isomorphic homogeneous Riemannian space $\left(M^{\prime}=G^{\prime} / H^{\prime}, g^{M^{\prime}}\right)$ is a GO-space. Also a direct product of Riemannian manifolds is a manifold with homogeneous geodesics if and only if each factor is a manifold with homogeneous geodesics. 


\section{Totally geodesic orbits in a homogeneous Riemannian space}

In this section we deal with totally geodesic submanifolds of compact homogeneous Riemannian spaces. This is a useful tool for study of GO-spaces due to the following

Proposition 1 ([3, Theorem 11]). Every closed totally geodesic submanifold of a Riemannian manifold with homogeneous geodesics is a manifold with homogeneous geodesics.

Let $\left(M=G / H, g^{M}\right)$ be a compact Riemannian homogeneous space with the reductive decomposition (1).

Definition 3. A subspace $\mathfrak{p} \subset \mathfrak{m}$ is called totally geodesic if it is the tangent space at $o$ of a totally geodesic orbit $K o \subset G / H=M$ of a subgroup $K \subset G$.

Proposition 2. A subspace $\mathfrak{p} \subset \mathfrak{m}$ is totally geodesic if and only if the following two conditions hold:

a) $\mathfrak{p}$ generates a subalgebra of the form $\mathfrak{k}=\mathfrak{h}^{\prime}+\mathfrak{p}$, where $\mathfrak{h}^{\prime}$ is a subalgebra of $\mathfrak{h}$;

b) the endomorphism $\operatorname{ad}_{Z}^{\mathfrak{p}} \in \operatorname{End}(\mathfrak{p})$ for $Z \in \mathfrak{p}^{\perp}$ is g-skew-symmetric or, equivalently,

$$
U(\mathfrak{p}, \mathfrak{p}) \subset \mathfrak{p} .
$$

Proof. If $\mathfrak{p}$ is the tangent space of the orbit $K o=K / H^{\prime}$, then $\operatorname{Lie}(K)=\mathfrak{k}=\mathfrak{h}^{\prime}+\mathfrak{p}$, where $\mathfrak{h}^{\prime}=\operatorname{Lie}\left(H^{\prime}\right)$ is a subalgebra of $\mathfrak{h}$. Moreover, the formulas (4) and (5) imply $U(\mathfrak{p}, \mathfrak{p}) \subset \mathfrak{p}$. Conversely, the conditions $a$ ) and $b$ ) imply that $\mathfrak{p}$ is the tangent space of the totally geodesic orbit $K o$ of the subgroup $K$ generated by the subalgebra $\mathfrak{k}$.

\section{Corollary 1.}

i) A subspace $\mathfrak{p} \subset \mathfrak{m}$ is totally geodesic if a) holds and $A \mathfrak{p}=\mathfrak{p}$.

ii) If a totally geodesic subspace $\mathfrak{p}$ is $\operatorname{ad}_{\mathfrak{h}}$-invariant and A-invariant, then

$$
\left[\mathfrak{h}+\mathfrak{p}, \mathfrak{p}^{\perp}\right] \subset \mathfrak{p}^{\perp}
$$

Proof. $i)$ Assume that $A \mathfrak{p}=\mathfrak{p}$. Then $A \mathfrak{p}^{\perp}=\mathfrak{p}^{\perp}$ and $\left\langle\mathfrak{p}, \mathfrak{p}^{\perp}\right\rangle=0$. From $i$ ) and $A \mathfrak{p}=\mathfrak{p}$ we get $\langle Z,[X, A X]\rangle=0$ for any $X \in \mathfrak{p}$ and $Z \in \mathfrak{p}^{\perp}$. This implies

$$
\begin{aligned}
0=\langle[Z, X], A X\rangle & =\left\langle[Z, X]_{\mathfrak{m}}, A X\right\rangle=\left([Z, X]_{\mathfrak{m}}, X\right) \\
& =\left([Z, X]_{\mathfrak{p}}, X\right)=(U(X, X), Z) .
\end{aligned}
$$

ii) follows from the fact that the endomorphisms $\operatorname{ad}_{\mathfrak{h}+\mathfrak{p}}$ are $b$-skew-symmetric and preserves the subspace $\mathfrak{p}$. Hence, they preserve its $b$-orthogonal complement $\mathfrak{p}^{\perp}$.

Corollary 2. Let $(M=G / H, g)$ be a compact Riemannian homogeneous space and $K$ a connected subgroup of $G$. The orbit $P=K o=K / H^{\prime}$ is a totally geodesic submanifold if and only if the Lie algebra $\mathfrak{k}$ is consistent with the reductive decomposition (1) (that is $\mathfrak{k}=\mathfrak{k} \cap \mathfrak{h}+\mathfrak{k} \cap \mathfrak{m}=\mathfrak{h}^{\prime}+\mathfrak{p}$ ) and

$$
U(\mathfrak{p}, \mathfrak{p}) \subset \mathfrak{p}
$$

or, equivalently, the endomorphisms $\operatorname{ad}_{Z}^{\mathfrak{p}} \in \operatorname{End}(\mathfrak{p}), Z \in \mathfrak{p}^{\perp}$ are g-skew-symmetric. 


\section{Properties of GO-spaces}

Lemma 3. Let $\left(M=G / H, g^{M}\right)$ be a GO-space with the reductive decomposition (1) and $\mathfrak{m}=$ $\mathfrak{p}+\mathfrak{q}$ a g-orthogonal $\mathrm{Ad}_{H}$-invariant decomposition. Then

$$
U(\mathfrak{p}, \mathfrak{p}) \subset \mathfrak{p}, \quad U(\mathfrak{q}, \mathfrak{q}) \subset \mathfrak{q}
$$

and the endomorphisms $\operatorname{ad}_{\mathfrak{p}}^{\mathfrak{q}}$, $\operatorname{ad}_{\mathfrak{q}}^{\mathfrak{p}}$ are skew-symmetric.

Proof. For $X \in \mathfrak{p}, Y \in \mathfrak{q}$ we have

$$
0=\left(\left[Y+H_{Y}, X\right]_{\mathfrak{m}}, Y\right)=-\left(\operatorname{ad}_{X} Y, Y\right)=-(U(Y, Y), X),
$$

where $H_{Y}$ is as in Lemma 2. This shows that $\operatorname{ad}_{X}^{\mathfrak{q}}$ is skew-symmetric and $U(\mathfrak{q}, \mathfrak{q}) \subset \mathfrak{q}$.

Lemma 3 together with Proposition 2 implies

Proposition 3. Let $\left(M=G / H, g^{M}\right)$ be a GO-space with the reductive decomposition (1). Then any connected subgroup $K \subset G$ which contains $H$ has the totally geodesic orbit $P=K o=K / H$ which is GO-space (with respect to the induced metric). Moreover, if the space $\mathfrak{p}:=\mathfrak{k} \cap \mathfrak{m}$ is A-invariant, then

$$
\left[\mathfrak{k}, \mathfrak{m}^{\perp}\right] \subset \mathfrak{m}^{\perp}
$$

and the metric $\bar{g}:=\left.g\right|_{\mathfrak{p}^{\perp}}$ is $\mathrm{Ad}_{K}$-invariant and defines an invariant GO-metric $g^{N}$ on the homogeneous manifolds $N=G / K$. The projection $\pi: G / H \rightarrow G / K$ is a Riemannian submersion with totally geodesic fibers such that the fibers and the base are GO-spaces.

Proof. The first claim follows from Lemma 3, Lemma 2 and Proposition 2. If $A \mathfrak{p}=\mathfrak{p}$, then $\mathfrak{m}=$ $\mathfrak{p}+\mathfrak{p}^{\perp}$ is a $b$-orthogonal decomposition and since the metric $b$ is $\operatorname{Ad}_{G}$-invariant, $\operatorname{Ad}_{K} \mathfrak{p}^{\perp}=\mathfrak{p}^{\perp}$. Then Lemma 3 shows that the metric $\left.g\right|_{\mathfrak{p}^{\perp}}$ is $\mathrm{Ad}_{K^{-}}$invariant and defines an invariant metric $g^{N}$ on $N=G / K$ such that $N$ becomes GO-space.

Note that a subgroup $K \supset H$ is compatible with any invariant metric on $G / H$ if $\operatorname{Ad}_{H^{-}}$ modules $\mathfrak{p}$ and $\mathfrak{m} / \mathfrak{p}$ are strictly disjoint. This remark implies

Proposition 4. Let $(M=G / H, g)$ be a compact homogeneous Riemannian space. Then the connected normalizer $N_{0}(Z)$ of a central subgroup $Z$ of $H$ and the connected normalizer $N_{0}(H)$ are subgroups consistent with any invariant metric on $M$.

Proposition 5. Let $\left(M=G / H, g^{M}\right)$ be a compact GO-space with metric endomorphism A.

i) Let $X, Y \in \mathfrak{m}$ be eigenvectors of the metric endomorphism A with different eigenvalues $\lambda, \mu$. Then

$$
[X, Y]=\frac{\lambda}{\lambda-\mu}[H, X]+\frac{\mu}{\lambda-\mu}[H, Y]
$$

for some $H \in \mathfrak{h}$.

ii) Assume that the vectors $X, Y$ belong to the $\lambda$-eigenspace $\mathfrak{m}_{\lambda}$ of $A$ and $X$ is $g$-orthogonal to the subspace $[\mathfrak{h}, Y]$. Then

$$
[X, Y] \in \mathfrak{h}+\mathfrak{m}_{\lambda}
$$


Proof. $i$ ) Let $X, Y \in \mathfrak{m}$ be eigenvectors of $A$ with different eigenvalues $\lambda, \mu$ and $H=H_{X+Y} \in \mathfrak{h}$ the element defined in Lemma 2. Then

$$
\begin{aligned}
{[H+X+Y, A(X+Y)] } & =[H+X+Y, \lambda X+\mu Y] \\
& =\lambda[H, X]+\mu[H, Y]+(\mu-\lambda)[X, Y] \in \mathfrak{h} .
\end{aligned}
$$

By Lemma 1, $[H, X],[H, Y],[X, Y] \in \mathfrak{m}$ and the right hand side is zero.

ii) Assume now that $X, Y \in \mathfrak{m}_{\lambda}$ satisfy conditions $\left.i i\right)$ and $Z$ is an eigenvector of $A$ with an eigenvalue $\mu \neq \lambda$. Then we have

$$
\begin{aligned}
\left([X, Y]_{\mathfrak{m}}, Z\right) & =\mu\langle[X, Y], Z\rangle=\mu\langle X,[Y, Z]\rangle=\frac{\mu}{\lambda}\left(X,[Y, Z]_{\mathfrak{m}}\right) \\
& =\frac{\mu}{\lambda}\left(X, \frac{\lambda}{\lambda-\mu}[H, Y]+\frac{\mu}{\lambda-\mu}[H, Z]\right)=0 .
\end{aligned}
$$

This shows that $[X, Y] \in \mathfrak{h}+\mathfrak{m}_{\lambda}$.

Corollary 3. Let $\left(M=G / H, g^{M}\right)$ be a compact GO-space with the reductive decomposition (1) and metric endomorphism $A$ and

$$
\mathfrak{m}=\mathfrak{m}_{1}+\cdots+\mathfrak{m}_{k}
$$

the A-eigenspace decomposition such that $\left.A\right|_{\mathfrak{m}_{i}}=\lambda_{i} 1_{\mathfrak{m}_{i}}$. Then for any $\operatorname{Ad}_{H}$-submodules $\mathfrak{p}_{i} \subset \mathfrak{m}_{i}$, $\mathfrak{p}_{j} \subset \mathfrak{m}_{j}, i \neq j$, we have

$$
\left[\mathfrak{p}_{i}, \mathfrak{p}_{j}\right] \subset \mathfrak{p}_{i}+\mathfrak{p}_{j}
$$

Moreover, if $\mathfrak{p}, \mathfrak{p}^{\prime}$ are g-orthogonal $\mathrm{Ad}_{H}$-submodules of $\mathfrak{m}_{i}$ then

$$
\left[\mathfrak{p}, \mathfrak{p}^{\prime}\right] \subset \mathfrak{h}+\mathfrak{m}_{i} \text {. }
$$

\section{Some applications}

\subsection{A sufficient condition for non-existence of GO-metric}

Here we consider some applications of results of the previous section.

Definition 4. Let $\left(M=G / H, g^{M}\right)$ be a compact homogeneous Riemannian space. A connected closed Lie subgroup $K \subset G$ which contains $H$ is called compatible with the metric $g^{M}$ if the subspace $\mathfrak{p}=\mathfrak{k} \cap \mathfrak{m}$ of $\mathfrak{m}$ is invariant under the metric endomorphism $A$.

Let $K, K^{\prime}$ be two subgroups of $G$ which are compatible with the metric of a homogeneous Riemannian space $\left(M=G / H, g^{M}\right)$. Then we can decompose the space $\mathfrak{m}$ into a $g$-orthogonal sum of $A$-invariant $\operatorname{Ad}_{H}$-modules

$$
\mathfrak{m}=\mathfrak{q}+\mathfrak{p}_{1}+\mathfrak{p}_{2}+\mathfrak{n}
$$

where

$$
\mathfrak{q}=\mathfrak{p} \cap \mathfrak{p}^{\prime}, \quad \mathfrak{p}=\mathfrak{k} \cap \mathfrak{m}=\mathfrak{q}+\mathfrak{p}_{1}, \quad \mathfrak{p}^{\prime}=\mathfrak{k}^{\prime} \cap \mathfrak{m}=\mathfrak{q}+\mathfrak{p}_{2}
$$

and $\mathfrak{n}$ is the orthogonal complement to

$$
\mathfrak{p}+\mathfrak{p}^{\prime}=\mathfrak{q}+\mathfrak{p}_{1}+\mathfrak{p}_{2}
$$

in $\mathfrak{m}$. 
Proposition 6. Let $\left(M=G / H, g^{M}\right)$ be a homogeneous Riemannian space, $K, K^{\prime}$ two subgroups of $G$ which are compatible with $g^{M}$ and $(7)$ the associated decomposition as above. Then $\mathfrak{p}_{1}, \mathfrak{p}_{2}, \mathfrak{n}$ are $\operatorname{Ad}_{\widetilde{H}}$-modules, where $\widetilde{H}=K \cap K^{\prime}$ is the Lie group with the Lie algebra $\widetilde{\mathfrak{h}}=\mathfrak{h}+\mathfrak{q}$, and

$$
\left[\mathfrak{p}_{1}, \mathfrak{p}_{2}\right] \subset \mathfrak{n} .
$$

Moreover, if $\left(M=G / H, g^{M}\right)$ is a GO-space, then the restriction $A^{\widetilde{\mathfrak{m}}}$ of the metric endomorphism to $\widetilde{\mathfrak{m}}=\mathfrak{p}_{1}+\mathfrak{p}_{2}+\mathfrak{n}$ commutes with $\left.\operatorname{Ad}_{\widetilde{H}}\right|_{\widetilde{\mathfrak{m}}}$ and for any $\widetilde{H}$-irreducible submodules $\mathfrak{p}_{1}^{\prime} \subset \mathfrak{p}_{1}$, and $\mathfrak{p}_{2}^{\prime} \subset \mathfrak{p}_{2}$ such that

$$
\left[\mathfrak{p}_{1}^{\prime}, \mathfrak{p}_{2}^{\prime}\right] \neq 0,
$$

the metric endomorphism $A$ is a scalar on the space

$$
\mathfrak{p}_{1}^{\prime}+\mathfrak{p}_{2}^{\prime}+\left[\mathfrak{p}_{1}^{\prime}, \mathfrak{p}_{2}^{\prime}\right] .
$$

Proof. Since the decomposition (7) is b-orthogonal, we conclude that it is $\operatorname{Ad}_{\widetilde{H}}$-invariant and

$$
\begin{aligned}
& {\left[\mathfrak{p}, \mathfrak{p}^{\perp}\right]=\left[\mathfrak{p}, \mathfrak{p}_{2}+\mathfrak{n}\right] \subset \mathfrak{p}_{2}+\mathfrak{n},} \\
& {\left[\mathfrak{p}^{\prime},\left(\mathfrak{p}^{\prime}\right)^{\perp}\right]=\left[\mathfrak{p}^{\prime}, \mathfrak{p}_{1}+\mathfrak{n}\right] \subset \mathfrak{p}_{1}+\mathfrak{n},}
\end{aligned}
$$

by Proposition 2. This implies

$$
\left[\mathfrak{p}_{1}, \mathfrak{p}_{2}\right] \subset \mathfrak{n} .
$$

If $\left(M, g^{M}\right)$ is a GO-space, then by Proposition 3 the metric endomorphism $A^{\widetilde{\mathfrak{m}}}$ is $\widetilde{H}$-invariant. If modules $\mathfrak{p}_{1}^{\prime}, \mathfrak{p}_{2}^{\prime}$ belong to $A$-eigenspaces with different eigenvalues, then by Corollary 3 ,

$$
\left[\mathfrak{p}_{1}^{\prime}, \mathfrak{p}_{2}^{\prime}\right] \subset \mathfrak{p}_{1}+\mathfrak{p}_{2}
$$

Together with the previous inclusion, it implies $\left[\mathfrak{p}_{1}^{\prime}, \mathfrak{p}_{2}^{\prime}\right]=0$. If these modules belong to the same eigenspace $\mathfrak{m}_{\lambda}$, then by Corollary 3 ,

$$
\left[\mathfrak{p}_{1}^{\prime}, \mathfrak{p}_{2}^{\prime}\right] \subset \mathfrak{m}_{\lambda} .
$$

As a corollary, we get the following sufficient condition that a homogeneous manifold $M=$ $G / H$ does not admit a proper GO-metric.

Proposition 7. Let $M=G / H$ be a homogeneous space of a compact group $G$ with the reductive decomposition $\mathfrak{g}=\mathfrak{h}+\mathfrak{m}$. Assume that the Lie algebra $\mathfrak{g}$ has two subalgebras $\mathfrak{k}=\mathfrak{h}+\mathfrak{p}, \mathfrak{k}^{\prime}=\mathfrak{h}+\mathfrak{p}^{\prime}$ which contain $\mathfrak{h}$ and generate $\mathfrak{g}$. Let

$$
\mathfrak{m}=\mathfrak{q}+\mathfrak{p}_{1}+\mathfrak{p}_{2}+\mathfrak{n}, \quad \mathfrak{q}=\mathfrak{p} \cap \mathfrak{p}^{\prime}
$$

be the associated b-orthogonal decomposition. Assume that there is no commuting $\operatorname{ad}_{\mathfrak{h}+\mathfrak{q}}$ submodules of $\mathfrak{p}_{1}$ and $\mathfrak{p}_{2}$. Then for any GO-metric, defined by an operator $A$ which preserves this decomposition, $A$ is a scalar operator on $\mathfrak{p}_{1}+\mathfrak{p}_{2}+\mathfrak{n}$. In particular, if $\mathfrak{q}$ is trivial and $\mathrm{Ad}_{H}-$ modules $\mathfrak{p}_{1}, \mathfrak{p}_{2}, \mathfrak{n}$ are strictly non-equivalent, then the only GO-metric on $M$ is the normal metric.

Proof. Let $A$ be an operator on $\mathfrak{m}$ which preserves the decomposition (8) and defines a GOmetric. Then by Proposition 6,

$$
\left.A\right|_{\mathfrak{p}_{1}+\mathfrak{p}_{2}+\left[\mathfrak{p}_{1}, \mathfrak{p}_{2}\right]}=\lambda \cdot \mathbf{1}
$$

for some $\lambda$. Now $\mathfrak{p}_{1}$ and $\left[\mathfrak{p}_{1}, \mathfrak{p}_{2}\right] \subset \mathfrak{n}$ are two $g$-orthogonal submodules of the $A$-eigenspace $\mathfrak{m}_{\lambda}$. Applying Corollary 3, we conclude that

$$
\left[\mathfrak{p}_{1},\left[\mathfrak{p}_{1}, \mathfrak{p}_{2}\right]\right] \subset \mathfrak{m}_{\lambda} .
$$

Iterating this process, we prove that

$$
\mathfrak{n}=\left[\mathfrak{p}_{1}, \mathfrak{p}_{2}\right]+\left[\mathfrak{p}_{1},\left[\mathfrak{p}_{1}, \mathfrak{p}_{2}\right]\right]_{\mathfrak{n}}+\left[\mathfrak{p}_{2},\left[\mathfrak{p}_{1}, \mathfrak{p}_{2}\right]\right]_{\mathfrak{n}}+\cdots \subset \mathfrak{m}_{\lambda}
$$

and $A=\lambda \cdot \mathbf{1}$ on $\mathfrak{p}_{1}+\mathfrak{p}_{2}+\mathfrak{n}$. 


\subsection{A sufficient condition for existence of GO-metric}

Lemma 4. Let $M=G / H$ be a homogeneous space of a compact Lie group with a reductive decomposition $\mathfrak{g}=\mathfrak{h}+\mathfrak{m}$. Assume that $\mathrm{Ad}_{H}$-module $\mathfrak{m}$ has a decomposition

$$
\mathfrak{m}=\mathfrak{m}_{1}+\cdots+\mathfrak{m}_{k}
$$

into invariant submodules, such that for any $i<j$

$$
\left[\mathfrak{m}_{i}, \mathfrak{m}_{j}\right]=0
$$

or this condition valid with one exception $(i, j)=(1,2)$ and in this case

$$
\left[\mathfrak{m}_{1}, \mathfrak{m}_{2}\right] \subset \mathfrak{m}_{2}
$$

and for any $X \in \mathfrak{m}_{1}, Y \in \mathfrak{m}_{2}$ there is $H \in \mathfrak{h}$ such that $\operatorname{ad}_{H} Y=\operatorname{ad}_{X} Y$ and

$$
\operatorname{ad}_{H}\left(\mathfrak{m}_{1}+\mathfrak{m}_{3}+\cdots+\mathfrak{m}_{k}\right)=0 .
$$

Then any metric endomorphism of the form $A=\sum x_{i} \cdot \mathbf{1}_{\mathfrak{m}_{i}}$ defines a GO-metric on $M$.

Proof. Under the assumptions of lemma, for $H \in \mathfrak{h}$ and $X_{i} \in \mathfrak{m}_{i}$ we have

$$
\begin{aligned}
{\left[H+\sum X_{i}, \sum x_{i} X_{i}\right] } & =\sum_{i<j}\left(x_{j}-x_{i}\right)\left[X_{i}, X_{j}\right]+\sum_{i} x_{i} \operatorname{ad}_{H} X_{i} \\
& =\left(x_{2}-x_{1}\right) \operatorname{ad}_{X_{1}} X_{2}+x_{2} \operatorname{ad}_{H} X_{2}+\operatorname{ad}_{H}\left(x_{1} X_{1}+\sum_{k \geq 3} x_{k} X_{k}\right) .
\end{aligned}
$$

The right-hand side is zero if $H$ is chosen as in the lemma (where $Y=x_{2} X_{2}$ and $X=\left(x_{1}-\right.$ $\left.x_{2}\right) X_{1}$ ). Now, it suffices to apply Lemma 2 .

Example 1. The homogeneous space $M=S U_{p+q} / S U_{p} \times S U_{q}$ is a GO-space with respect to any invariant metric.

We have the reductive decomposition

$$
\mathfrak{s u}_{p+q}=\mathfrak{h}+\mathfrak{m}=\left(\mathfrak{s u}_{p}+\mathfrak{s u}_{q}\right)+(\mathbb{R} a+\mathfrak{p}),
$$

where $\mathfrak{p} \simeq \mathbb{C}^{p} \otimes \mathbb{C}^{q}$ and $\left.\operatorname{ad}_{a}\right|_{\mathfrak{p}}=i \cdot \mathbf{1}_{\mathfrak{p}}$. Any metric endomorphism $A=\lambda \cdot \mathbf{1}_{\mathbb{R} a}+\mu \cdot \mathbf{1}_{\mathfrak{p}}$ defines a GO-metric by above lemma since for any $X \in \mathfrak{p}$ there is $H \in \mathfrak{h}$ such that $\operatorname{ad}_{H} X=i X$. Note that for $p \neq q$ these manifolds are weakly symmetric spaces [26].

\subsection{GO-metrics on a compact group $G$}

Proposition 8. A compact Lie group $G$ with a left-invariant metric $g$ is a GO-space if and only if the corresponding Euclidean metric $(\cdot, \cdot)$ on the Lie algebra $\mathfrak{g}$ is bi-invariant.

Proof. The condition that $(G, g)$ is a GO-space can be written as

$$
0=(X,[X, Y])=-\left(\operatorname{ad}_{Y} X, X\right)=0 .
$$

This shows that the metric $(\cdot, \cdot)$ is bi-invariant.

Note that a compact Lie group $G$ can admit a non-bi-invariant left-invariant metrics $g$ with homogeneous geodesics. But the corresponding GO-space will have the form $L / H$ where the group $L$ will contain $G$ as a proper subgroup. See [11] for details. 


\section{Homogeneous GO-spaces with positive Euler characteristic}

\subsection{Basic facts about homogeneous manifolds of positive Euler characteristic}

Here we recall some properties of homogeneous spaces with positive Euler characteristic (see, for example, [21] or [4]). A homogeneous space $M=G / H$ of a compact connected Lie group $G$ has positive Euler characteristic $\chi(M)>0$ if and only if the stabilizer $H$ has maximal rank $(\operatorname{rk}(H)=\operatorname{rk}(G))$.

If the group $G$ acts on $M$ almost effectively, then it is semisimple and the universal covering $\widetilde{M}=\widetilde{G} / \widetilde{H}$ is a direct product

$$
\widetilde{M}=G_{1} / H_{1} \times \cdots \times G_{k} / H_{k},
$$

where $\widetilde{G}=G_{1} \times G_{2} \times \cdots \times G_{k}$ is the decomposition of the group $\widetilde{G}$ (which is a covering of $G$ ) into a direct product of simple factors and $H_{i}=\widetilde{H} \cap G_{i}$.

Any invariant metric $g^{M}$ on $M$ defines an invariant metric $g^{\widetilde{M}}$ on $\widetilde{M}$ and the homogeneous Riemannian space $\left(\widetilde{M}=\widetilde{G} / \widetilde{H}, g^{\widetilde{M}}\right)$ is a direct product of homogeneous Riemannian spaces $\left(M_{i}=G_{i} / H_{i}, g^{M_{i}}\right), i=1, \ldots, k$, of simple compact Lie groups $G_{i}$, see [19]. We have

Proposition 9 ([19]). A compact almost effective homogeneous Riemannian space ( $M=$ $\left.G / H, g^{M}\right)$ of positive Euler characteristic is irreducible if and only if the group $G$ is simple. If the group $G$ acts effectively on $M$, it has trivial center.

This proposition shows that a simply connected compact GO-space $\left(M=G / H, g^{M}\right)$ of positive Euler characteristic is a direct product of simply connected GO-spaces $\left(M_{i}=G_{i} / H_{i}, g^{M_{i}}\right)$ of simple Lie groups with positive Euler characteristic. So it is sufficient to classify simply connected GO-spaces of a simple compact Lie group with positive Euler characteristic.

A description of homogeneous spaces $G / H$ of positive Euler characteristic reduces to description of connected subgroups $H$ of maximal rank of $G$ or equivalently, subalgebras of maximal rank of a simple compact Lie algebra $\mathfrak{g}$, see [9] and also Section 8.10 in [25]. An important subclass of compact homogeneous spaces of positive Euler characteristic consists of flag manifolds. They are described as adjoint orbits $M=\operatorname{Ad}_{G} x$ of a compact connected semisimple Lie group $G$ or, in other terms as quotients $M=G / H$ of $G$ by the centrelizer $H=Z_{G}(T)$ of a non-trivial torus $T \subset G$.

Note that every compact naturally reductive homogeneous Riemannian space of positive Euler characteristic is necessarily normal homogeneous with respect to some transitive semisimple isometry group [4].

\subsection{The main theorem}

Let $G$ be a simple compact connected Lie group, $H \subset K \subset G$ its closed connected subgroups. We denote by $b=\langle\cdot, \cdot\rangle$ the minus Killing form on the Lie algebra $\mathfrak{g}$ and consider the following $b$-orthogonal decomposition

$$
\mathfrak{g}=\mathfrak{h} \oplus \mathfrak{m}=\mathfrak{h} \oplus \mathfrak{m}_{1} \oplus \mathfrak{m}_{2},
$$

where

$$
\mathfrak{k}=\mathfrak{h} \oplus \mathfrak{m}_{2}
$$

is the Lie algebra of the group $K$. Obviously, $\left[\mathfrak{m}_{2}, \mathfrak{m}_{1}\right] \subset \mathfrak{m}_{1}$. Let $g^{M}=g_{x_{1}, x_{2}}$ be a $G$-invariant Riemannian metric on $M=G / H$, generated by the Euclidean metric $g=(\cdot, \cdot)$ on $\mathfrak{m}$ of the form

$$
g=x_{1} \cdot b_{\mathfrak{m}_{1}}+x_{2} \cdot b_{\mathfrak{m}_{2}},
$$


where $x_{1}$ and $x_{2}$ are positive numbers, or, equivalently, by the metric endomorphism

$$
A=x_{1} \cdot \mathbf{1}_{\mathfrak{m}_{1}}+x_{2} \cdot \mathbf{1}_{\mathfrak{m}_{2}} .
$$

We consider two examples of such homogeneous Riemannian spaces $\left(M=G / H, g_{x_{1}, x_{2}}\right)$ :

a) $(G, K, H)=(S O(2 n+1), U(n), S O(2 n)), n \geq 2$. The group $G=S O(2 n+1)$ acts transitively on the symmetric space $\operatorname{Com}\left(\mathbb{R}^{2 n+2}\right)=S O(2 n+2) / U(n)$ of complex structures in $\mathbb{R}^{2 n+2}$ with stabilizer $H=U(n)$, see [15]. So we can identify $M=G / H$ with this symmetric space, but the metric $g_{x_{1}, x_{2}}$ is not $S O(2 n+2)$-invariant if $x_{2} \neq 2 x_{1}$ [17].

b) $(G, K, H)=(S p(n), S p(1) \cdot S p(n-1), U(1) \cdot S p(n-1)), n \geq 2$. The group $G=S p(n)$ acts transitively on the projective space $\mathbb{C} P^{2 n-1}=S U(2 n+2) / U(2 n+1)$ with stabilizer $H=U(1) \cdot S p(n-1)$. So we can identify $M=G / H$ with $\mathbb{C} P^{2 n-1}$, but the metric $g_{x_{1}, x_{2}}$ is not $S U(2 n+2)$-invariant if $x_{2} \neq 2 x_{1}$, see $[15,17]$.

Now we can state the main theorem about compact GO-spaces of positive Euler characteristic.

Theorem 1. Let $\left(M=G / H, g^{M}\right)$ is a simply connected proper GO-space with positive Euler characteristic and simple compact Lie group $G$. Then $M=G / H=S O(2 n+1) / U(n), n \geq$ 2 , or $G / H=S p(n) / U(1) \times S p(n-1), n \geq 2$, and $g^{M}=g_{x_{1}, x_{2}}$ is any $G$-invariant metric which is not $G$-normal homogeneous. The metric $g^{M}$ is $G$-normal homogeneous (respectively, symmetric) when $x_{2}=x_{1}$ (respectively, $x_{2}=2 x_{1}$ ). Moreover, these homogeneous spaces are weakly symmetric flag manifolds.

The non-symmetric metrics $g_{x_{1}, x_{2}}$ have $G$ as the full connected isometry group of the considered GO-spaces $\left(M=G / H, g_{x_{1}, x_{2}}\right)$, see discussion in $[17,21,1]$. The claim that all these homogeneous Riemannian spaces are weakly symmetric spaces was proved in [27]. Note also that Theorem 1 allows to simplify some arguments in the paper [3].

\subsection{Proof of the main theorem}

Using results from [1] and [3], we reduce the proof to a description of some special decompositions of the root system of the Lie algebra $\mathfrak{g}$ of the isometry group $G$.

Let $M=G / H$ be a homogeneous space of a compact simple Lie group of positive characteristic and

$$
\mathfrak{g}=\mathfrak{h}+\mathfrak{m}
$$

associated reductive decomposition. The subgroup $H$ contains a maximal torus $T$ of $G$. We consider the root space decomposition

$$
\mathfrak{g}^{\mathbb{C}}=\mathfrak{t}^{\mathbb{C}}+\sum_{\alpha \in R} \mathfrak{g}_{\alpha}
$$

of the complexification $\mathfrak{g}^{\mathbb{C}}$ of the Lie algebra $\mathfrak{g}$, where $\mathfrak{t}^{\mathbb{C}}$ is the Cartan subalgebra associated with $T$ and $R$ is the root system.

For any subset $P \subset R$ we denote by

$$
\mathfrak{g}(P)=\sum_{\alpha \in P} \mathfrak{g}_{\alpha}
$$

the subspace spanned by corresponding root space $\mathfrak{g}_{\alpha}$. Then $H$-module $\mathfrak{m}^{\mathbb{C}}$ is decomposed into a direct sum

$$
\mathfrak{m}^{\mathbb{C}}=\mathfrak{g}\left(R_{1}\right)+\cdots+\mathfrak{g}\left(R_{k}\right)
$$


of disjoint submodules, where $R=R_{1} \cup \cdots \cup R_{k}$ is a disjoint decomposition of $R$ and subsets $R_{i}$ are symmetric, i.e. $-R_{i}=R_{i}$. Moreover, real $H$-modules $\mathfrak{g} \cap \mathfrak{g}\left(R_{i}\right)$ are irreducible. Any invariant metric on $M$ is defined by the metric endomorphism $A$ on $\mathfrak{m}$ whose extension to $\mathfrak{m}^{\mathbb{C}}$ has the form

$$
A=\operatorname{diag}\left(x_{1} \cdot \mathbf{1}_{\mathfrak{p}_{1}}, \ldots, x_{\ell} \cdot \mathbf{1}_{\mathfrak{p}_{\ell}}\right)
$$

where $x_{i}$ are arbitrary positive numbers, $x_{i} \neq x_{j}$ and $\mathfrak{p}_{i}$ is a direct sum of modules $\mathfrak{g}\left(R_{m}\right)$.

We will assume that $A$ is not a scalar operator (i.e. $\ell>1$ ) and it defines an invariant metric with homogeneous geodesics. We say that a root $\alpha$ corresponds to eigenvalue $x_{i}$ of $A$ if $\mathfrak{g}_{\alpha} \subset \mathfrak{p}_{i}$.

Lemma 5. There are two roots $\alpha, \beta$ which correspond to different eigenvalues of $A$ such that $\alpha+\beta$ is a root.

Proof. If it is not the case, $\left[\mathfrak{p}_{1}, \mathfrak{p}_{i}\right]=0$ for $i \neq 1$ and $\mathfrak{g}_{1}=\mathfrak{p}_{1}+\left[\mathfrak{p}_{1}, \mathfrak{p}_{1}\right]$ would be a proper ideal of a simple Lie algebra $\mathfrak{g}$.

Now, consider the roots $\alpha$ and $\beta$ as in the previous lemma. Since $R(\alpha, \beta):=R \cap \operatorname{span}\{\alpha, \beta\}$ is a rank 2 root system, we can always choose roots $\alpha, \beta \in R$ which form a basis of the root system $R(\alpha, \beta)$. Then the subalgebra

$$
\mathfrak{g}_{\alpha, \beta}:=\mathfrak{t}^{\mathbb{C}}+\sum_{\gamma \in R(\alpha, \beta)} \mathfrak{g}_{\gamma}
$$

of $\mathfrak{g}^{\mathbb{C}}$ is the centralizer of the subalgebra $\mathfrak{t}^{\prime}=\operatorname{ker} \alpha \cap \operatorname{ker} \beta \subset \mathfrak{t}^{\mathbb{C}}$.

Then the orbit $G_{\alpha, \beta} O \subset M$ of the corresponding subgroup $G_{\alpha, \beta}=T^{\prime} \cdot G_{\alpha, \beta}^{\prime} \subset G$ is a totally geodesic submanifold (see Corollary 2), hence a proper GO-space with the effective action of the rank two simple group $G_{\alpha, \beta}^{\prime}$ associated with the root system $R(\alpha, \beta)$ (see Proposition 1). Note that it has positive Euler characteristic since the stabilizer of the point $o$ contains the two-dimensional torus generated by vectors $H_{\alpha}, H_{\beta} \in \mathfrak{t}^{\mathbb{C}}$ associated with roots $\alpha, \beta$. Recall that $H_{\alpha}=\frac{2}{\langle\alpha, \alpha\rangle} b^{-1} \cdot \alpha$.

Proposition 10. Every proper $G O$-space $\left(M=G / H, g^{M}\right)$ with positive Euler characteristic of a simple group $G$ of rank 2 is locally isometric to the manifold $M=S O(5) / U(2)$ with the metric defined by the metric endomorphism

$$
A=x_{1} \cdot \mathbf{1}_{\mathfrak{g}\left(R^{s}\right)}+x_{2} \cdot \mathbf{1}_{\mathfrak{g}\left(R^{\ell}\right)}, \quad x_{1} \neq x_{2}>0
$$

where

$$
R^{s}=\left\{ \pm \epsilon_{1}, \pm \epsilon_{2}\right\}, \quad R^{\ell}=\left\{ \pm \epsilon_{1} \pm \epsilon_{2}\right\},
$$

are the sets of short and, respectively, long roots of the Lie algebra $\mathfrak{s o}(5)$. We may assume also that

$$
\mathfrak{m}^{\mathbb{C}}=\mathfrak{g}\left(R^{s} \cup\left\{\epsilon_{1}+\epsilon_{2}\right\}\right) \quad \text { and } \quad \mathfrak{h}^{\mathbb{C}}=\mathfrak{t}^{\mathbb{C}}+\mathfrak{g}_{\epsilon_{1}-\epsilon_{2}} .
$$

Proof. Proof of this proposition follows from results of the papers [1] and [3]. Indeed, the group $G$ has the Lie algebra $\mathfrak{g}$ isomorphic to $s u(3)=A_{2}$, so(5) $=s p(2)=B_{2}=C_{2}$ or $g_{2}$. Since the universal Riemannian covering of a GO-space is a GO-space (Lemma 2), we may assume without loss of generality that $G / H$ is simply connected.

If $\mathfrak{g}=s u(3)$, then $G / H=S U(3) / S(U(2) \times U(1))$ (a symmetric space) or $G / H=S U(3) / T^{2}$, where $T^{2}$ is a maximal torus in $S U(3)$. Both these spaces are flag manifolds, and results of [1] show that any GO-metric on these spaces is $S U(3)$-normal homogeneous. 
If $\mathfrak{g}=s o(5)=s p(2)$, then $(\mathfrak{g}, \mathfrak{h})=\left(s o(5), \mathbb{R}^{2}\right),(\mathfrak{g}, \mathfrak{h})=\left(s o(5), \mathbb{R} \oplus s u(2)_{l}\right),(\mathfrak{g}, \mathfrak{h})=(s o(5), \mathbb{R} \oplus$ $\left.s u(2)_{s}\right)$, or $(\mathfrak{g}, \mathfrak{h})=\left(s o(5), s u(2)_{l} \oplus s u(2)_{l}\right)$, where $s u(2)_{l}$, (respectively, $\left.s u(2)_{s}\right)$ stands for a threedimensional subalgebras generated by all long (respectively, short) roots of $\mathfrak{g}$. The last pair corresponds to the irreducible symmetric space $S O(5) / S O(4)$, which admits no non-normal invariant metric. All other spaces are flag manifolds. Results of [1] implies that the only possible pair is $(\mathfrak{g}, \mathfrak{h})=\left(s o(5), \mathbb{R} \oplus s u(2)_{l}\right)$, which corresponds to the space $S O(5) / U(2)=$ $S p(2) / U(1) \cdot S p(1)$.

For $\mathfrak{g}=g_{2}$ the statement of proposition is proved in [3, Proposition 23].

Corollary 4. Let $G$ be a simple compact Lie group and $M=G / H$ a proper $G O$-space with positive Euler characteristic. Then the root system $R$ of the complex Lie algebra $\mathfrak{g}^{\mathbb{C}}$ admits a disjoint decomposition

$$
R=R_{0} \cup R_{1} \cup R_{2}
$$

where $R_{0}$ is the root system of the complexified stability subalgebra $\mathfrak{h}^{\mathbb{C}}$, with the following properties:

i) If $\alpha \in R_{1}, \beta \in R_{2}$ and $\alpha+\beta \in R$ then $\alpha-\beta \in R$ and the rank 2 root system $R(\alpha, \beta)$ has type $B_{2}=C_{2}$.

ii) Moreover, if $\alpha, \beta$ is a basis of $R(\alpha, \beta)$ (that is $\langle\alpha, \beta\rangle<0$ ), then one of the roots $\alpha, \beta$ is short and the other is long and one of the long roots $\alpha \pm \beta$ belongs to $R_{0}$ and second one belongs to $R_{1} \cup R_{2}$.

iii) If both roots $\alpha, \beta$ are short, then one of the long roots $\alpha \pm \beta$ belongs to $R_{0}$ and the other belongs to $R_{1} \cup R_{2}$.

iv) If $\alpha \in R_{1}$ and $\beta \in R_{2}$ are long roots, then $\alpha \pm \beta \notin R$.

We will call a decomposition with the above properties a special decomposition. Corollary 4 implies

Corollary 5. There is no proper GO-spaces of positive Euler characteristic with simple isometry group $G=S U(n), S O(2 n), E_{6}, E_{7}, E_{8}$ (these are all simple Lie algebras with all roots of the same length (simply-laced root system)).

Corollary 6 ([3, Proposition 23]). Any GO-space $(G / H, \mu)$ of positive Euler characteristic with $G=G_{2}$ is normal homogeneous.

Now, we describe all special decompositions of the root systems of types $B_{n}, C_{n}, F_{4}$. We will use notation from [13] for root systems and simple roots.

Lemma 6. The root system

$$
R\left(F_{4}\right)=\left\{ \pm \epsilon_{i}, 1 / 2\left( \pm \epsilon_{1}+\mp \epsilon_{2} \pm \epsilon_{3}+ \pm \epsilon_{4}, \pm \epsilon_{i} \pm \epsilon_{j}\right), i, j=1,2,3,4, i \neq j\right\}
$$

does not admit a special decomposition.

Proof. Assume that such a decomposition exists. Then we can choose roots $\alpha \in R_{1}, \beta \in R_{2}$ such that $\alpha \pm \beta$ is a root. Then $\alpha, \beta$ has different length and we may assume that $|\alpha|<|\beta|$ and $\langle\alpha, \beta\rangle<0$. Then we can include $\alpha, \beta$ into a system of simple $\operatorname{roots} \delta, \alpha, \beta, \gamma$, see [13]. Since all such systems are conjugated, we may assume that $\alpha=\epsilon_{4}, \beta=-\epsilon_{4}+\epsilon_{3}$, see [13]. Then we get contradiction, since $\alpha-\beta$ is not a root. 
Now we describe two special decompositions for the root systems

$$
R\left(B_{n}\right)=\left\{ \pm \epsilon_{i}, \pm \epsilon_{i} \pm \epsilon_{j}, i, j=1, \ldots, n\right\}
$$

and

$$
R\left(C_{n}\right)=\left\{ \pm 2 \epsilon_{i}, \pm \epsilon_{i} \pm \epsilon_{j}, i, j=1, \ldots, n\right\}
$$

of types $B_{n}$ and $C_{n}$. Note that in both cases $R_{A}=\left\{ \pm\left(\epsilon_{i}-\epsilon_{j}\right)\right\}$ is a closed subsystem. We set $R_{A}^{+}=\left\{ \pm\left(\epsilon_{i}+\epsilon_{j}\right)\right\}$.

We denote by $R^{+}$the standard subsystem of positive roots of a root system $R$ and by $R^{s}$ and $R^{\ell}$ the subset of short and, respectively, long roots of $R$. Then there is a special decomposition $R=R_{0} \cup R_{1} \cup R_{2}$ of the systems $R\left(B_{n}\right), R\left(C_{n}\right)$ which we call the standard decomposition:

$$
\begin{aligned}
& R\left(B_{n}\right)=R_{A} \cup R^{s} \cup R_{A}^{+}, \\
& R\left(C_{n}\right)=R_{A} \cup R^{\ell} \cup R_{A}^{+} .
\end{aligned}
$$

These decompositions define the following reductive decompositions of the homogeneous spaces $S O(2 n+1) / U(n)$ and $S p(n) / U(n)$ :

$$
\begin{aligned}
& \mathfrak{s o}(2 n+1)=\mathfrak{h}+\left(\mathfrak{m}_{\mathbf{1}}+\mathfrak{m}_{2}\right)=\mathfrak{g}\left(R_{A}\right)+\left(\mathfrak{g}\left(R^{s}\right)+\mathfrak{g}\left(R_{A}^{+}\right)\right), \\
& \mathfrak{s p}(n)=\mathfrak{h}+\left(\mathfrak{m}_{\mathbf{L}}+\mathfrak{m}_{2}\right)=\mathfrak{g}\left(R_{A}\right)+\left(\mathfrak{g}\left(R^{\ell}\right)+\mathfrak{g}\left(R_{A}^{+}\right)\right),
\end{aligned}
$$

where $\mathfrak{m}_{1}, \mathfrak{m}_{2}$ are irreducible submodules of $\mathfrak{m}$. It is known [1] that any metric endomorphism $A=\operatorname{diag}\left(x_{1} \cdot \mathbf{1}_{\mathfrak{m}_{1}}, x_{2} \cdot \mathbf{1}_{\mathfrak{m}_{2}}\right)$ defines a metric with homogeneous geodesics on the corresponding manifold $M=G / H$ (see a discussion before the statement of Theorem 1). Now, the proof of Theorem 1 follows from the following proposition.

Proposition 11. Any special decomposition of the root systems $R_{B}, R_{C}$ is conjugated to the standard one.

Proof. We give a proof of this proposition for $R\left(B_{n}\right)$. The proof for $R\left(C_{n}\right)$ is similar.

Let

$$
R\left(B_{n}\right)=R_{0} \cup R_{1} \cup R_{2}
$$

be a special decomposition of $R\left(B_{n}\right)$. We may assume that there are roots $\alpha \in R_{1}$ and $\beta \in R_{2}$ with $\langle\alpha, \beta\rangle<0$ and $|\alpha|<|\beta|$. Then we can include $\alpha, \beta$ into a system of simple roots, which, without loss of generality, can be written as

$$
\alpha_{1}=\epsilon_{1}-\epsilon_{2}, \ldots, \epsilon_{n-2}-\epsilon_{n-1}, \epsilon_{n-1}+\epsilon_{n}=\beta,-\epsilon_{n}=\alpha .
$$

Then $\left(\epsilon_{n-1}-\epsilon_{n}\right) \in R_{0}$. We need the following lemma.

Lemma 7. Let $R\left(B_{n}\right)=R_{0} \cup R_{1} \cup R_{2}$ be a special decomposition as above, $V^{\prime}=\epsilon_{n}^{\perp}$ the orthogonal complement of the vector $\epsilon_{n}$ and $R\left(B_{n-1}\right)=R^{\prime}:=R \cap V^{\prime}$ the root system induced in the hyperspace $V^{\prime}$. Then the induced decomposition $R^{\prime}=R_{0}^{\prime} \cup R_{1}^{\prime} \cup R_{2}^{\prime}$, where $R_{i}^{\prime}:=R_{i} \cap V^{\prime}$, is a special decomposition.

Proof. It is sufficient to check that subsets $R_{1}^{\prime}, R_{2}^{\prime}$ are not empty.

We say that two roots $\gamma, \delta$ are $R_{0}$-equivalent $(\gamma \sim \delta)$ if their difference belongs to $R_{0}$. The equivalent roots belong to the same component $R_{i}$. The root $\epsilon_{n-1}=\epsilon_{n}-\left(\epsilon_{n-1}-\epsilon_{n}\right)$ is $R_{0}$-equivalent to $\alpha=\epsilon_{n}$. Hence it belongs to $R_{1}$. 
We say that a pair of roots $\gamma, \delta$ with $\langle\gamma, \delta\rangle<0$ is special if one of the roots belongs to $R_{1}$ and another to $R_{2}$. Then they have different length (say, $|\gamma|<|\delta|$ ). Moreover, the root $\gamma+\delta$ is short and it belongs to the same part $R_{i}, i=1,2$ as the short root $\delta$ and the root $2 \gamma+\delta$ is long and it belongs to $R_{0}$.

Consider the roots $\sigma_{ \pm}= \pm \epsilon_{n-2}+\epsilon_{n-1}$. They have negative scalar product with $\epsilon_{n-1} \in R_{1}$ and $\beta=\epsilon_{n-1}+\epsilon_{n} \in R_{2}$. They can not belong to $R_{1}$ since then we get a special pair $\delta_{ \pm}, \beta$ which consists of long roots. They both can not belong to $R_{0}$ since otherwise the root $\epsilon_{n-2} \sim \epsilon_{n-1} \in R_{1}$ and $\pm \epsilon_{n-2}+\epsilon_{n} \sim \epsilon_{n-1}+\epsilon_{n} \in R_{2}$ and we get a special pair

$$
\gamma=\epsilon_{n-2} \in R_{1}, \quad \delta=-\epsilon_{n-2}+\epsilon_{n} \in R_{2},
$$

such that $2 \gamma+\delta \in R_{0}$, which is impossible. We conclude that one of the roots $\sigma_{ \pm}= \pm \epsilon_{n-2}+$ $\epsilon_{n-1} \in R^{\prime}$ must belongs to $R_{2}$. Since the root $\epsilon_{n-1} \in R^{\prime}$ belongs to $R_{1}$, the lemma is proved.

Now we prove the proposition by induction on $n$. The claim is true for $n=2$ by Proposition 10. Assume that it is true for $R(B(n-1))$ and let $R\left(B_{n}\right)=R_{0} \cup R_{1} \cup R_{2}$ be a special decomposition as above. By lemma, the decomposition $R^{\prime}=R_{0}^{\prime} \cup R_{1}^{\prime} \cup R_{2}^{\prime}$, indiced in the hyperplane $V^{\prime}=e_{n} \perp$, is a special decomposition. By inductive hypothesis we may assume that it has the standard form:

$$
R_{0}=\left\{ \pm\left(\epsilon_{i}-\epsilon_{j}\right)\right\}, \quad R_{1}=\left\{ \pm \epsilon_{i}\right\}, \quad R_{2}=\left\{ \pm\left(\epsilon_{i}+\epsilon_{j}\right), i, j=1, \ldots, n-1\right\} .
$$

This implies that the initial decomposition is also standard.

\section{Acknowledgements}

The first author was partially supported by the Royal Society (Travel Grant 2007/R3). The second author was partially supported by the State Maintenance Program for the Leading Scientific Schools of the Russian Federation (grant NSH-5682.2008.1). We are grateful to all referees, whose comments and suggestions permit us to improve the presentation of this article.

\section{References}

[1] Alekseevsky D.V., Arvanitoyeorgos A., Riemannian flag manifolds with homogeneous geodesics, Trans. Amer. Math. Soc. 359 (2007), 3769-3789.

[2] Akhiezer D.N., Vinberg E.B., Weakly symmetric spaces and spherical varieties, Transform. Groups 4 (1999), $3-24$.

[3] Berestovskii V.N., Nikonorov Yu.G., On $\delta$-homogeneous Riemannian manifolds, Differential Geom. Appl. 26 (2008), 514-535, math.DG/0611557.

[4] Berestovskii V.N., Nikonorov Yu.G., On $\delta$-homogeneous Riemannian manifolds. II, Siber. Math. J. 50 (2009), $214-222$.

[5] Berestovskii V.N., Nikitenko E.V., Nikonorov Yu.G., The classification of $\delta$-homogeneous Riemannian manifolds with positive Euler characteristic, arXiv:0903:0457.

[6] Berger M., Les variétés riemanniennes homogènes normales à courbure strictement positive, Ann. Scuola Norm. Sup. Pisa (3) 15 (1961), 179-246.

[7] Berndt J., Kowalski O., Vanhecke L., Geodesics in weakly symmetric spaces, Ann. Global Anal. Geom. 15 (1997), 153-156.

[8] Besse A.L., Einstein manifolds, Springer-Verlag, Berlin, 1987.

[9] Borel A., de Siebenthal J., Les sous-groups fermés de rang maximum des groups de Lie clos, Comment. Math. Helv. 23 (1949), 200-221.

[10] Cartan É., Sur une classe remarquable d'espaces de Riemann, Bull. Soc. Math. France 54 (1926), $214-264$. Cartan É., Sur une classe remarquable d'espaces de Riemann. II, Bull. Soc. Math. France 55 (1927), 114-134. 
[11] D'Atri J.E., Ziller W., Naturally reductive metrics and Einstein metrics on compact Lie groups, Mem. Amer. Math. Soc. 18 (1979), no. 215, 1-72.

[12] Dušek Z., Kowalski O., Nikčević S., New examples of Riemannian g.o. manifolds in dimension 7, Differential Geom. Appl. 21 (2004), 65-78.

[13] Gorbatzevich V.V., Onishchik A.L., Vinberg E.B., Lie groups and Lie algebras. III. Structure of Lie groups and Lie algebras, Encyclopaedia of Mathematical Sciences, Vol. 41, Springer-Verlag, Berlin, 1994.

[14] Gordon C.S., Homogeneous Riemannian manifolds whose geodesics are orbits, in Topics in Geometry: in Memory of Joseph D'Atri, Progr. Nonlinear Differential Equations Appl., Vol. 20, Birkhäuser Boston, Boston, MA, 1996, 155-174.

[15] Helgason S., Differential geometry and symmetric spaces, Pure and Applied Mathematics, Vol. 12, Academic Press, New York - London, 1962.

[16] Kaplan A., On the geometry of groups of Heisenberg type, Bull. London Math. Soc. 15 (1990), 32-42.

[17] Kerr M.M., Some new homogeneous Einstein metrics on symmetric spaces, Trans. Amer. Math. Soc. 348 (1996), 153-171.

[18] Kobayashi S., Nomizu K., Foundations of differential geometry, John Wiley \& Sons, New York, Vol. I, 1963, Vol. II, 1969.

[19] Kostant B., On holonomy and homogeneous spaces, Nagoya Math. J. 12 (1957), 31-54.

[20] Kowalski O., Vanhecke L., Riemannian manifolds with homogeneous geodesics, Boll. Un. Mat. Ital. B (7) 5 (1991), 189-246.

[21] Onishchik A.L., Topology of transitive transformation groups, Johann Ambrosius Barth Verlag GmbH, Leipzig, 1994.

[22] Selberg A., Harmonic analysis and discontinuous groups in weakly symmetric Riemannian spaces, with applications to Dirichlet series, J. Indian Math. Soc. (N.S.) 20 (1956), 47-87.

[23] Tamaru H., Riemannin G.O. spaces fibered over irreducible symmetric spaces, Osaka J. Math. 15 (1998), 835-851.

[24] Tamaru H., Riemannin geodesic orbit space metrics on fiber bundles, Algebra Groups Geom. 36 (1999) 835-851.

[25] Wolf J.A., Spaces of constant curvature, Publish or Perish, Inc., Houston, TX, 1984.

[26] Wolf J.A., Harmonic analysis on commutative spaces, Mathematical Surveys and Monographs, Vol. 142, American Mathematical Society, Providence, RI, 2007.

[27] Ziller W., Weakly symmetric spaces, in Topics in Geometry: in Memory of Joseph D'Atri, Progr. Nonlinear Differential Equations Appl., Vol. 20, Birkhäuser Boston, Boston, MA, 1996, 355-368.

[28] Yakimova O.S., Weakly symmetric Riemannian manifolds with a reductive isometry group, Sb. Math. 195 (2004), 599-614. 